\title{
Linx
}

Revue des linguistes de l'université Paris X Nanterre

9 | 1997

Émile Benveniste. Vingt ans après

\section{Sur la terminologie de Benveniste}

\section{Mohammad Djafar Moïnfar}

\section{(2) OpenEdition \\ Journals}

Édition électronique

URL : http://journals.openedition.org/linx/1085

DOI : 10.4000/linx.1085

ISSN : 2118-9692

\section{Éditeur}

Presses universitaires de Paris Nanterre

\section{Édition imprimée}

Date de publication : 1 avril 1997

Pagination : 365-374

ISSN : 0246-8743

\section{Référence électronique}

Mohammad Djafar Moïnfar, «Sur la terminologie de Benveniste », Linx [En ligne], 9| 1997, mis en ligne

le 25 juillet 2012, consulté le 19 avril 2019. URL : http://journals.openedition.org/linx/1085 ; DOI :

10.4000/linx.1085 


\title{
Sur la terminologie de Benveniste
}

\author{
Mohammad Djafar Moinfar
}
"...il est parfois utile de demander à l'évidence de se justifier."

E. Benveniste ${ }^{1}$

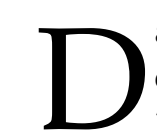

ans son article "Formes nouvelles de la composition nominale", publié en $1966^{2}$, en analysant le mot "microbe", E. Benveniste fait remarquer : "il est impossible de deviner les conditions dans lesquelles un néologisme a été créé ; il faut les découvrir à la source même, dans l'intention $\mathrm{du}$ créateur. Rien ne peut dispenser de cette recherche, qui est pour l'étymologiste une obligation."

C'est ce principe qui m'a guidé dans la conception d'un ouvrage, en chantier depuis un certain temps, sur la terminologie de Benveniste. Un travail que je considère comme un complément à sa bibliographie, que j'ai établie il y a vingt ans ${ }^{3}$, et qui a pour ambition d'offrir un instrument qui facilitera, je l'espère, les recherches concernant sa pensée et son œuvre. En outre, une telle monographie pourra contribuer à l'élaboration d'un dictionnaire complet de la linguistique, selon encore un principe énoncé par Benveniste, à la fin d'un compte rendu qu'il fait du dictionnaire de linguistique de Mario A. Pei et Frank Gaynor" : “Un vrai dictionnaire linguistique devrait être l'œuvre d'une

\footnotetext{
1 "De la subjectivité dans le langage", in Journal de Psychologie Normale et Pathologique, 55 (juilletseptembre 1958), 257-265 ; repris dans les Problèmes de linguistique générale 1, 258-266.

2 In Bulletin de la Société de Linguistique de Paris, tome 61 (1966), fasc. 1, pp. 82-95; repris dans les Problèmes de linguistique générale 2, 163-167.

3 Mohammad Djafar Moïnfar, Bibliographie des travaux d'Emile Benveniste, Paris, Société de Linguistique de Paris, 1975. (Extrait des Mélanges Linguistiques Offerts à Emile Benveniste). Dépositaire : Editions Peeters, B. P. 41, 3000 Louvain, Belgique.

${ }^{4}$ In Bulletin de Société de Linguistique de Paris, tome 51 (1955), fasc. 2, pp. 5-8 : Mario A. Pei et Frank Gaynor, Dictionary of Linguistics, New York, 1954.
} 


\section{Mohammad Djafar Mö̈nfar}

équipe travaillant sur une documentation considérable et avec un sens critique aiguisé."

Dans cette communication, je me permets de présenter quelques exemples typiques et significatifs de la terminologie de Benveniste et de mettre en évidence les critères qui gouvernent son choix.

Le premier travail scientifique d'Emile Benveniste, "Les futurs et subjonctifs sigmatiques du latin archaïque", est paru en 1922 dans le Bulletin de la Société de Linguistique de Paris, tome 23, fasc. 1 ( $\left.{ }^{\circ} 70\right)$, pp. 32-63. Mais son achèvement proprement dit doit être antérieur à 1921. Car, Benveniste, en examinant les types $\overline{a m} \overline{a s s} s \overline{0}$ et $f \bar{a} \bar{x} \overline{0}$, passe en revue les différents travaux déjà parus à leur sujet et indique, p. 52, "un opuscule récent de M. Pedersen, paru après l'achèvement du présent travail (Les formes sigmatiques $d u$ verbe latin et le problème du futur indo-européen, Copenhague, 1921) est venu affirmer, après les travaux cités p. 36, l'autonomie du futur indo-européen." Affirmant que c'est à l'intérieur même du latin que doivent être recherchés les éléments d'une explication, Benveniste considère, p. 53, que "Le latin a innové en utilisant ici d'une manière indépendante un procédé que nombre de langues ont employé et dont on n'a pas assez reconnu l'importance : la gémination expressive." Et puis, il donne la précision suivante concernant cette expression: "On conçoit, en effet, que si l'on appuie avec insistance sur une idée ou sur l'aspect de cette idée qui affecte le sentiment, le besoin inconscient d'être expressif ait pour effet de donner une particulière intensité à l'un des éléments caractéristiques du mot, - en général une consonne -, et, en l'espèce, de la géminer. Dans le vocabulaire, de pareils faits de redoublement ont été bien souvent constatés, mais non point toujours ramenés au principe dont ils dérivent." 5

Dans son article "L'anaphorique prussien din et le système des démonstratifs indo-européen", publié en 1933 dans Studi Baltici, vol. 3, pp. 121130, E. Benveniste, après un examen approfondi des données, formule, p. 124, le principe selon lequel "à chaque thème indo-européen de démonstratif, caractérisé par une valeur forte, une forme tonique, la voyelle thématique, la soumission au cas, au genre et au nombre, peut s'opposer un anaphorique caractérisé par une valeur faible, la position enclitique, la voyelle - $i$ - et une forme fixe indifférente au genre et au nombre." Et aussitôt après, il donne la précision suivante: "Ces propriétés structurelles et syntaxiques de l'anaphorique sont données dans la définition de l'anaphore. Entre la deixis et l'anaphore, la différence n'est pas de degré ni dans l'insistance plus ou moins forte apportée à l'indication. Chacun des deux procédés dénote une conception

\footnotetext{
5 J. Marouzeau, dans son Lexique de la terminologie linguistique, $3^{\circ}$ éd., 1951, p. 100, ne donne que cette courte définition: "De la gémination organique ou naturelle on distingue parfois une gémination expressive, p. ex. celle de la syllabe dans les formations du type bêbête, fifille..."
} 
distincte de la détermination déictique. La deixis établit une relation variable entre celui qui parle ou celui à qui on parle ou celui dont on parle et un objet qui doit lui aussi être caractérisé par sa position lato sensu. L'instrument de la deixis, le démonstratif, est soumis à un grand nombre de contraintes pour exprimer avec la netteté désirable le rapport en question. Il doit faire savoir immédiatement non seulement la proximité plus ou moins grande de l'objet et par rapport à qui, mais encore toutes les déterminations de cas, de genre et de nombre que porte l'appellatif lui-même. La deixis est une réelle qualification, qui intègre l'objet dans un système de positions; et l'on sait que plus on remonte aux origines d'une mentalité, plus le nombre et la complexité de ces relations vont croissant. Au contraire l'anaphorique, renvoyant par définition à ce qui est connu, n'appelle aucune précision supplémentaire de genre ou de nombre. Aucune ambiguïté ne peut se produire. L'objet venant d'être explicitement désigné, il suffit d'une indication mécanique pour le faire à nouveau surgir dans l'esprit. L'anaphore est un procédé de rappel, et l'anaphorique, un outil grammatical... L'anaphorique est au démonstratif ce que le démonstratif est à l'objet montré : il ne renvoie pas à la chose, mais à la notion antérieurement formulée de cette chose. Il est le signe d'un signe. A cette unicité du concept correspondra une forme unique, qui sera nécessairement au singulier et nécessairement à un cas complément, car un emploi comme sujet en ferait un véritable démonstratif. L'anaphorique sera donc au cas objet par excellence, l'accusatif, et se comportera comme un complément, quels que soient le genre et le nombre requis."

Emile Benveniste dans une étude qui, selon lui, "ne veut être qu'une opération de contrôle", publiée, en 1935, dans les Mémoires de la Société de Linguistique de Paris, tome 23, sixième fascicule, pp. 393-405, sous le titre "Les absolutifs avestiques", recherche, "sans préoccupation doctrinale ce que valent philologiquement dans leur contexte les formations avestiques dites $\mathrm{d}^{\prime}$ absolutif, et particulièrement celles en -am, où Bartholomae a pensé retrouver l'équivalent des absolutifs sanskrits en -am."

Benveniste examine d'abord le seul exemple sans préverbe que Bartholomae a relevé dans les textes anciens : jum ( = jívam) dans Yt V, 63, yezi jum frapayemi aoi zam "şi j'arrive vivant sur la terre". Il considère qu'il y a ici abus de mot ; "si l'on doit appeler absolutif tout accusatif nominal apposé à un verbe, il faudra renoncer à une définition rigoureuse." Et il ajoute la précision suivante : "Seule mérite le nom d'absolutif une forme liée morphologiquement et syntaxiquement au verbe et annexant à la proposition principale une détermination verbale, c'est-à-dire une forme caractérisée comme verbale par sa structure et par son sens; or ̌va- simple adjectif, contredit sous ce double rapport la notion d'absolutif."

Aussi, en examinant cas par cas les exemples présentés, il conclut que "partout où il ne s'agit pas de fautes, on a affaire à une dérivation de l'adjectif ou de participe survenant sporadiquement à basse époque. L'avestique n'offre 


\section{Mohammad Djafar Mö̈nfar}

rien de comparable aux absolutifs sanskrits en -am. Ces derniers, ne trouvant plus d'appui en iranien, doivent être rapportés à une innovation indienne..."

Dans son article "La philosophie analytique et le langage", publié en 19636, (p. 274), E. Benveniste, rejetant Austin qui classe comme performatifs les énoncés conçus à l'impératif précise ainsi : "Nous n'avons pas ici affaire à une modalité spécifique du discours ; l'impératif n'est pas dénotatif et ne vise pas à communiquer un contenu, mais se caractérise comme pragmatique et vise à agir sur l'auditeur, à lui intimer un comportement. L'impératif n'est pas un temps verbal; il ne comporte ni marque temporelle ni référence personnelle. C'est le sémantème nu employé comme forme jussive avec une intonation spécifique. On voit donc qu'un impératif n'équivaut pas à un énoncé performatif, pour cette raison qu'il n'est ni énoncé ni performatif. Il n'est pas énoncé, puisqu'il ne sert pas à construire une proposition à verbe personnel; et il n'est pas performatif, du fait qu'il ne dénomme pas l'acte de parole à performer.... l'impératif produit un comportement, mais l'énoncé performatif est l'acte même qu'il dénomme et qui dénomme son performateur. Nous repousserons donc toute identification de l'un à l'autre."

E. Benveniste commence ainsi son article "De la subjectivité dans le langage", publié en $1958^{7}$ : "Si le langage est, comme on dit, instrument de communication, à quoi doit-il cette propriété ? La question peut surprendre, comme tout ce qui a l'air de mettre en question l'évidence, mais il est parfois utile de demander à l'évidence de se justifier." Et plus loin (p. 259) il signale que "En réalité la comparaison du langage avec un instrument, et il faut bien que ce soit avec un instrument matériel pour que la comparaison soit simplement intelligible, doit nous remplir de méfiance, comme toute notion simpliste au sujet du langage. Parler d'instrument, c'est mettre en opposition l'homme et la nature. La pioche, la flèche, la roue ne sont pas dans la nature. Ce sont des fabrications. Le langage est dans la nature de l'homme, qui ne l'a pas fabriqué... Tous les caractères du langage, sa nature immatérielle, son fonctionnement symbolique, son agencement articulé, le fait qu'il a un contenu, suffisent déjà à rendre suspecte cette assimilation à un instrument, qui tend à dissocier de l'homme la propriété du langage. Assurément, dans la pratique quotidienne, le va-et-vient de la parole suggère un échange, donc une "chose" que nous échangerions, elle semble donc assumer une fonction instrumentale ou véhiculaire que nous sommes prompts à hypostasier en un "objet". Mais, encore une fois, ce rôle revient à la parole."

\footnotetext{
${ }^{6}$ In Les Etudes philosophiques, $\mathrm{n}^{\circ} 1$ (janvier-mars 1963, 3-11) ; repris dans les Problèmes de linguistique générale 1, 267-276, auxquels, comme dans les autres cas identiques, par commodité, je renvoie.

7 In Journal de Psychologie Normale et Pathologique, 58 (1958), pp. 257-265; repris dans les Problèmes de linguistique générale 1, 258-266.
} 
Dans l'article "Fondements syntaxiques de la composition nominale", publié en $1967^{8}$, où tous les types de composition nominale sont décrits et analysés, E. Benveniste souligne que aussi bien dans la pratique descriptive que dans la théorie des classes de formes, on a toujours considéré que la composition nominale relève de la morphologie. Sans contester que les particularités formelles des mots composés intéressent effectivement la morphologie nominale, il fait remarquer que (p. 145) "la considération morphologique laisse sans réponse et à vrai dire ne permet même pas de poser le problème fondamental : quelle est la fonction des composés ? Qu'est-ce que les rend possibles et pourquoi sont-ils nécessaires ? Dans une langue consistant en signe simples, l'existence d'unités faites de deux signes conjoints invite à se demander où est la source commune des composés d'où provient la diversité de leurs formes."

Pour répondre à cette question il faut, à son avis, "envisager les composés non plus comme des espèces morphologiques, mais comme des organisations syntaxiques. La composition nominale est une micro-syntaxe. Chaque type de composés est à étudier comme la transformation d'un type d'énoncé syntaxique libre." Or, de son examen, sous cette considération, des principales classes de composés, il ressort (p. 160), que "On ne peut donc plus expliquer la création des composés par la simple jonction immédiate de deux signes antérieurs... C'est le modèle syntaxique qui crée la possibilité du composé morphologique et qui le produit par transformation. La proposition, en ses différents types, émerge ainsi dans la zone nominale.

Dès lors, il faut reconnaître aux composés une situation particulière. En général on les range, avec les dérivés, dans la "formation des noms". Il faudrait plutôt les faire entrer dans un chapitre qui pourrait s'appeler "métamorphisme": nous entendons par là, dit Benveniste, le processus de transformation de certaines classes en d'autres."

Dans l'article "La subjectivité dans le langage", déjà mentionné, remarquons (p. 265) le passage suivant: "On discernera mieux encore la nature de cette "subjectivité" en considérant les effets de sens que produit le changement des personnes dans certains verbes de parole. Ce sont des verbes qui dénotent par leur sens un acte individuel de portée sociale : jurer, promettre, certifier... Dans les conditions sociales où la langue s'exerce, les actes dénotés par ces verbes sont regardés comme contraignants. Or ici, la différence entre l'énonciation "subjective" et l'énonciation "non subjective" apparaît en pleine lumière, dès qu'on s'est avisé de la nature de l'opposition entre les "personnes" du verbe... Or je jure est une forme de valeur singulière, en ce qu'elle place sur celui qui s'énonce je la réalité du serment. Cette énonciation est un accomplissement: "jurer" consiste précisément en l'énonciation je jure, par quoi Ego est lié.

\footnotetext{
8 In Bulletin de la Société de Linguistique de Paris, tome 62 (1967), fasc. 1, pp. 15-21; repris dans les
} Problèmes de linguistique générale 2, 145-162. 


\section{Mohammad Djafar Moïnfar}

L'énonciation je jure est l'acte même qui m'engage, non la description de l'acte que j'accomplis. En disant je promets, je garantis, je promets et je garantis effectivement. Les conséquences (sociale, juridique, etc.) de mon serment, de ma promesse, se déroulent à partir de l'instance de discours contenant je jure, je promets. L'énonciation s'identifie avec l'acte même."

On a bien compris qu'il s'agit ici du performatif, sans qu'il soit expressément nommé. En 1962, paraissent les actes d'un colloque organisé à Royaumont, consacré à la philosophie analytique ${ }^{9}$, dans lesquels figure, en particulier, l'article de J. L. Austin "Performatif-constatif". Cette publication incite $\mathrm{E}$. Benveniste à examiner de nouveau, et cette fois-ci en détail et le nommant, le performatif, précisant ses propres vues et à les confronter avec celles d'Austin, dans un article intitulé "La philosophie analytique et le langage", publié en $1963^{10}$. Sur le plan de la terminologie, il nous fait remarquer (p. 270, note 4): "Puisque performance est déjà entré dans l'usage, il n'y aura pas de difficulté à y introduire performatif au sens particulier qu'il a ici. On ne fait d'ailleurs que ramener en français une famille lexicale que l'anglais a pris à l'ancien français : perform vient de l'ancien français performer. Quant au terme constatif, il est régulièrement fait sur constat. Bien que constat soit étymologiquement le présent latin constat "il est constant", le français le traite comme un substantif de même série que résultat et le rattache ainsi à la famille de l'ancien verbe conster "être constant". Le rapport conster : constat est ainsi parallèle à résulter : résultat. Et de même que sur résultat, prédicat, on a fait résultatif, prédicatif, il sera licite de tirer de constat un adjectif constatif." Ensuite, en s'appuyant sur "la réalité des emplois", il propose (p. 271) la définition suivante : "les énoncés performatifs sont des énoncés où un verbe déclaratifjussif à la première personne du présent est construit avec un dictum. Ainsi j'ordonne (ou je commande, je décrète, etc.) que la population soit mobilisée, où le dictum est représenté par: la population est mobilisée. C'est bien un dictum, puisque l'énonciation expresse en est indispensable pour que le texte ait qualité de performatif." Il considère qu' "Une autre variété de tels énoncés est donnée par la construction du verbe avec un complément direct et un terme prédicatif : Je proclame $X$ directeur,..." Sont considérés aussi comme performatifs les énoncés qui ne sont qu' implicitement mis au compte de l'autorité habilitée à les produire, en usage aujourd'hui dans le formulaire officiel tel que $M$. X est

\footnotetext{
${ }^{9}$ La philosophie analytique. Paris, Les Editions de Minuit, 1962 ; réimp. 1990. (Cahiers de Royaumont, Philosophie, $\mathrm{n}^{\circ}$ IV).

10 In Les Etudes Philosophiques, n ${ }^{\circ} 1$ (janvier-mars 1963), pp. 3-11; repris dans les Problèmes de linguistique générale 1, pp. 267-276. Concernant les actes du colloque de Royaumont consacré à la philosophie analytique, Benveniste, p. 267, $\mathrm{n}^{\circ} 2$, trouve qu' "il est regrettable que la date à laquelle a eu lieu ce colloque n'apparaisse nulle part dans la publication." Il faut souligner que cet oubli est partiellement réparé dans la réimpression des actes du colloque. En effet, sur la dernière page de la couverture la date 1958 est donnée pour "cette première rencontre organisée en France, entre les principaux représentants des écoles anglaises et américaines de la philosophie analytique et ceux du continent."
} 
nommé ministre plénipotentiaire. - La chaire de botanique est déclarée vacante. Ils ne comportent pas de verbe déclaratif (Je décrète que...) et se réduisent au dictum, mais celui-ci est publié dans un recueil officiel, sous la signature de l'autorité, et parfois accompagné de l'incise par la présente. Ou encore le prononcé d'un dictum est rapporté impersonnellement et à la troisième personne : Il est décidé que... - Le Président de la République décrète que... Le changement consiste en une simple transposition. L'énoncé à la troisième personne peut toujours être reconverti en une première personne et reprendre sa forme typique. Ces énoncés performatifs sont produits dans le domaine des actes d'autorité. Un autre est celui où l'énoncé pose un engagement personnel pour celui qui l'énonce : Je jure..., je promets... Benveniste précise que "De toute manière, un énoncé performatif n'a de réalité que s'il est authentifié comme acte. Hors des circonstances qui le rendent performatif, un tel énoncé n'est plus rien... Un énoncé performatif qui n'est pas acte n'existe pas. Il n'a d'existence que comme acte d'autorité. Or, les actes d'autorité sont d'abord et toujours des énonciations proférées par ceux à qui appartient le droit de les énoncer. Cette condition de validité, relative à la personne énonçante et à la circonstance de l'énonciation, doit toujours être supposée remplie quand on traite du performatif. Là est le critère et non dans le choix des verbes. Un verbe quelconque de parole, même le plus commun de tous, le verbe dire, est apte à former un énoncé performatif si la formule: je dis que..., émise dans les conditions appropriées, crée une situation nouvelle... De cette condition en résulte une autre. L'énoncé performatif, étant un acte, a cette propriété d'être unique. Il ne peut être effectué que dans des circonstances particulières, une fois et une seule, à une date et à un lieu définis. Il n'a pas valeur de description ni de prescription, mais, encore une fois, d'accomplissement..."

Au commencement de l'article "Les verbes délocutifs", publié en 195811, E. Benveniste précise que le terme "délocutif" n'a pas encore cours en linguistique et qu'il l'introduit pour définir, et faire reconnaître dans sa particularité et dans sa généralité, une classe de verbes. Il s'agit des verbes qui sont dérivés de locutions. Or, de même qu'un verbe dit dénominatif dérive d'un nom, un verbe déverbatif dérive d'un verbe, les verbes en questions peuvent être appelés délocutifs. Après un examen des spécimens de ces verbes en plusieurs langues, par exemple, latin salutare "dire salut!", français remercier "dire merci", il conclut (p. 285) ${ }^{12}$ que "Le trait essentiel et signalétique d'un délocutif est qu'il est avec sa base nominale dans la relation "dire...", et non dans la relation "faire..." qui est propre au dénominatif. Ce n'est pas le caractère le moins instructif de cette classe de nous montrer un signe de la

\footnotetext{
11 In Studia Philologica et Litteraria in Honorem L. Spitzer, 1958, pp. 57-63 ; repris dans les Problèmes de linguistique générale 1, 277-285.

12 Peu importe que tel ou tel exemple de délocutif donné par Benveniste, en l'occurrence le latin salutare, soit contesté ; le procédé d'analyse proposé n'en souffre pas.
} 


\section{Mohammad Djafar Mö̈nfar}

langue dérivant d'une locution de discours et non d'un autre signe de la langue; de ce fait même, les délocutifs seront surtout, au moment où ils sont créés, des verbes dénotant des activités de discours. Leur structure aussi bien que les raisons qui les appellent à l'existence leur assignent une position toute particulière parmi les autres classes de dérivés verbaux."

Dans l'article consacré aux formes nouvelles de composition nominale (du français), publié dans le Bulletin de la Société de Linguistique de Paris, en $1966^{13}$, E. Benveniste donne la définition suivante pour la composition (p. 171) : "Il y a composition quand deux termes identifiables pour le locuteur se conjoignent en une unité nouvelle à signifié unique et constant." Par là, Il fait une distinction entre les composés et les unités nouvelles formées de syntagmes complexes comportant plus de deux éléments qu'il appelle Conglomérés. Les uns sont des syntagmes prédicatifs convertis en substantifs, tels que va-nu-pied, meurt-de-faim. Les autres, des locutions adverbiales où vivent des éléments archaïques, tels que dorénavant (= d'or en avant), désormais (= dès or mais) qui ne sont plus guère analysables, et jamais, qui ne l'est plus du tout. D'une manière générale, les conglomérés tendent à l'état de signe compact.

Ensuite, Benveniste insiste (p. 172) tout particulièrement sur un type de composition, non encore reconnu dans sa nature propre et n'ayant pas de statut défini. "Il consiste en un groupe entier de lexèmes, reliés par divers procédés, et formant une désignation constante et spécifique. On en trouve le noyau initial dans des exemples déjà anciens comme : pomme de terre, robe de chambre, clair de lune, plat à barbe. Le fait nouveau et important est qu'il prend aujourd'hui une extension considérable et qu'il est appelé à une productivité indéfinie : il est et sera la formation de base dans les nomenclatures techniques. Il suffit de mentionner des termes comme modulation de fréquence, avion à réaction, pour donner une idée du type, mais aussi pour montrer qu'il est construit sur un modèle qui n'est plus celui de la composition classique." La création d'un terme qui soit distinct de "composition" et de "syntagme", pour désigner ces grandes unités devenant nécessaire, Benveniste propose le terme

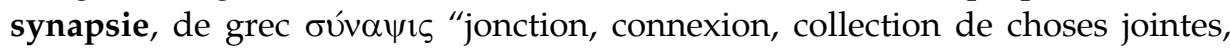

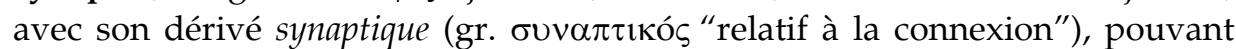
fournir, au besoin, des termes monosynaptique, disynaptique, polysynaptique, ou même synapter, synaptabe, etc.

Benveniste donne, en note, cette précision: "Il y a bien la synapse des neurophysiologistes, mais l'emploi est si différent qu'il ne fera pas confusion. Nous avons préféré pour l'adaption du mot grec en français la finale en -sie,

13 "Formes nouvelles de la composition nominale", BSL, 61 (1966),fasc. 1, 82-95 ; repris dans PLG 2, 163-175. 
d'après le modèle des mots grecs en -oisvenus à travers le latin : épilepsie, paralysie,-phylaxie,-syncrasie, poésie, etc."14

Quant aux traits qui caractérisent la synapsie, ce sont :

1) la nature syntaxique (non morphologique) de liaison entre les membres ;

2) l'emploi de joncteurs à cet effet, notamment de et à ;

3) l'ordre déterminé + déterminant des membres ;

4) leur forme lexicale pleine, et le choix libre de tout substantif ou adjectif ;

5) l'absence d'article devant le déterminant ;

6) la possibilité d'expansion pour l'un ou l'autre membre ;

7) le caractère unique et constant du signifié.

A titre d'exemple: soit garde-malade étant un composé, gardien d'asile et asile de nuit sont des synapsies ; gardien d'asile de nuit est une nouvelle synapsie à deux membres dont le premier simple, gardien, et le seconde synaptique luimême, asile de nuit, appelé dans le cas présent "subsynaptique".

Considérant ces quelques exemples, on peut conclure que : hormis le vocabulaire courant et sans problème en usage en linguistique, les termes techniques proprement dits utilisés par Benveniste sont de quatre sortes :

1 - Les termes déjà existants dont la définition est reprise, éclairée et développée, par exemple: gémination expressive, anaphorique, absolutif, impératif.

2 - Les termes déjà existants dans d'autres domaines que la linguistique auxquels il donne une nouvelle définition adaptée à ses besoins, par exemple : métamorphisme, congloméré.

3 -Les néologismes créés par d'autres chercheurs s'intéressant aux problèmes du langage, repris à son profit, par exemple : performatif.

4 - Les néologismes créés par lui-même, par exemple : délocutif, synapsie.

Autrement dit, malgré l'étendue impressionnante du domaine de ses recherches et l'immensité du champ de sa théorisation, Benveniste n'est pas partisan de la création coûte que coûte de nouveaux termes.

L'étude de son œuvre, qui s'étale sur une cinquantaine d'années, permet d'observer une constante remarquable du début à la fin à savoir: aller à l'essentiel, éviter le superflu, dans une optique pédagogique de la transmission du savoir. Ainsi, lorsque pour exprimer ses idées, Benveniste peut puiser dans la terminologie existante, il le fait; au besoin, en remodelant et en circonscrivant la définition et le champ d'application des termes. Mais quand,

14 Le Dictionnaire des termes techniques de médecine de Garnier et Delamare donne, p. 976 (18éd.,

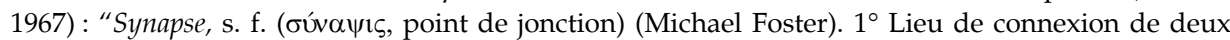
neurones - $2^{\circ}$ Syn. cénapse (Machebœuf). Nom parfois donné en chimie à certains corps complexes." 
Mohammad Djafar Moïnfar

et seulement quand, il le juge indispensable, il crée un nouveau terme et cela toujours en le justifiant. Qu'il s'agisse d'une de ces quatre sortes de termes, quelle qu'elle soit, la terminologie de Benveniste est toujours précise et limpide. Ce soucis va parfois si loin que cela peut paraître surfait; exemple, la définition donnée pour "instrument". Cependant, une relecture attentive et méditative conduit à constater la profondeur de sa réflexion. 